\title{
Endocannabinoids as markers of sperm quality: hot spots
}

\author{
Mauro Maccarrone ${ }^{1,2}$ * \\ ${ }^{1}$ Center of Integrated Research, Campus Bio-Medico University of Rome, Rome, Italy \\ ${ }^{2}$ European Center for Brain Research/Santa Lucia Foundation, Rome, Italy \\ *Correspondence: m.maccarrone@unicampus.it \\ Edited by: \\ Riccardo Pierantoni, Seconda Università di Napoli, Italy
}

Keywords: cholesterol, chromatin, epigenetic regulation, lipid rafts, receptors

Male reproductive health is under threat from a range of environmental and lifestyle assaults, including endocrine disrupters, toxic pollutants, and ionizing radiations, as well as lifestyle factors such as sexually transmitted infections, alcoholism, smoking, and anabolic steroid use. The latest potential hazard in our modern lifestyle is the use of plant-derived cannabinoids present in hashish and marijuana as recreational drugs, and more recently as therapeutic agents (1). In the last decade, a highly sophisticated endogenous cannabinoid system (ECS) has been discovered in mammals, where it regulates many physiological functions including human male reproduction (2-5). Here, I shall briefly discuss the activity of distinct ECS elements that can be useful to assess sperm function, and hence to potentially monitor sperm quality. Among others, these include the effect of type-1 cannabinoid receptor $\left(\mathrm{CB}_{1}\right)$ in regulating energy metabolism and motility of human sperm, and that of transient receptor potential vanilloid 1 (TRPV1) channels in controlling their fertilizing ability. Remarkably, both receptors share a common natural agonist, that is the endocannabinoid (eCB) $N$ arachidonoylethanolamine (anandamide, AEA); instead, another major eCB like 2arachidonoylglycerol (2-AG) can activate $\mathrm{CB}_{1}$, but is ineffective at TRPV1 receptors (6). The potential therapeutic exploitation of these ECS elements for the treatment of human infertility will be also addressed.

Human sperm express $\mathrm{CB}_{1}$, and its activation by AEA affects motility and acrosome reaction (AR). Both processes require energy, and a major role for glycolysis in supplying ATP for sperm motility has been recognized. Recently, human sperm exposure to methanandamide, a non-hydrolyzable analog of AEA, has been shown to significantly decrease mitochondrial transmembrane potential without triggering any mitochondria-dependent apoptotic death, and such an effect was prevented by the $\mathrm{CB}_{1}$ antagonist SR141716, but not by the $\mathrm{CB}_{2}$ antagonist SR144528, nor by the TRPV1 antagonist iodoresiniferatoxin (7). Interestingly, in the presence of glucose human sperm exposure to methanandamide for up to $18 \mathrm{~h}$ failed to affect sperm motility, that instead was dramatically reduced by the same substance under glycolysis blockage; again, the latter effect was prevented by SR141716 (7). Overall, $\mathrm{CB}_{1}$ activation induced a non-apoptotic decrease of mitochondrial potential, whose detrimental reflection on sperm motility could be revealed only when blocking glycolysis. These findings contribute to elucidate the relationship between $\mathrm{CB}_{1}$, energetic metabolism and mitochondria, an issue that appears relevant well beyond sperm biology. Indeed, mitochondrial $\mathrm{CB}_{1}$ activation has been recently reported to control energy metabolism in neurons (8), though the actual receptor localization on mitochondria remains controversial (9).

Another hot spot is the involvement of the AEA-binding TRPV1 receptor in human sperm fertilizing ability. Immunoreactivity for $\mathrm{CB}_{1}$ has been localized in the post-acrosomal region and in the midpiece of human sperm, whereas for TRPV1 it was restricted to the post-acrosomal region (10). Capsazepine (CPZ), a selective antagonist of TRPV1, was shown to inhibit progesterone (P)-enhanced sperm/oocyte fusion, as evaluated by the hamster egg penetration test. This inhibition was due to a reduction of the P-induced $\mathrm{AR}$ rate above that of spontaneous $\mathrm{AR}$, which was instead increased (10). Altogether, these data demonstrate that TRPV1 plays a keyrole in the human sperm fertilizing ability, by impacting on its fusion with the oocyte membrane. In line with this, a marked decrease of the ability of TRPV1 to bind its ligands has been shown in infertile versus fertile sperm, again supporting a major role for this ion channel in sperm functionality (11). On this basis, one might speculate that the reduction of AEA causes infertile sperm to lose their quiescent state and with that, the ability to prevent premature capacitation. This could then precipitate a premature AR, rendering that sperm infertile because of a reduced ability to penetrate an oocyte in vivo, or in assisted conception such as in in vitro fertilization (IVF) protocols. This hypothesis has recently found grounds through a clinical study performed on men affected by asthenozoospermia and oligoasthenoteratozoospermia (12). Indeed, AEA levels in seminal plasma were found to be halved in patients with respect to normal subjects $(\sim 0.08$ versus $\sim 0.20 \mathrm{nM})$. Remarkably, these differences in AEA content in men with different pathological semen subtypes were associated with poor semen quality, such as decreased sperm count and abnormal sperm motility, as well as with alterations of $\mathrm{CB}_{1}$ at transcriptional level (12). Therefore, evaluation of eCBs content in human sperm and/or in seminal plasma could be proposed as a novel diagnostic tool in reproductive medicine. In line with this, a marked reduction (down to $\sim 25 \%$ ) of both AEA and 2-AG content in seminal plasma from infertile men has been recently documented (11). Instead, no significant alterations were found in sperm from infertile versus fertile men, neither for AEA nor for 2-AG (11). Collectively, 
these data pinpoint eCBs (and AEA in particular) as new biomarkers to determine semen quality, thus opening new avenues for the treatment of infertility in humans.

Further points of interest in the regulation of sperm quality by ECS are related to the role of membrane properties and epigenetic control of chromatin activity.

Mammalian sperm become fertile after completing capacitation, a process associated with cholesterol loss and changes in the biophysical properties of the membranes, e.g., at the level of cholesterolrich microdomains termed lipid rafts (13). Membrane raft dynamics prepares the sperm to undergo AR, and in addition it may have a role in sperm-egg membrane interaction (14). Interestingly, $\mathrm{CB}_{1}$ and TRPV1 are affected by sperm membrane properties (15), and $\mathrm{CB}_{1}$ signal transduction in enhanced by lipid raft disruption in different neuronal and immune cells (16). In addition, the AEA congener $N$-palmitoylethanolamine (PEA), that has been shown in the male reproductive tract, modulates plasma membrane polarity with an effect on $\mathrm{Ca}^{2+}$ influx during the capacitation process (17). Remarkably, PEA might also affect some physiological sperm kinematic parameters (like sperm motility), thus impacting on the development of hyperactivation during capacitation, ultimately leading to idiopathic infertility (18). Taken together, further investigations into the contribution of sperm membrane lipid composition to the control of eCB signaling, and hence to its relevance for sperm quality and fertilizing ability, hold promise for a better design of preventive and/or therapeutic strategies against infertility. In this context, it remains to be assessed whether (and to what extent) sperm functionality might be affected by accumulation of AEA and congeners in intracellular stores called adiposomes (or lipid droplets), that are present in sperm (19), and are important for eCB signaling in different cell types $(20,21)$.

The last hot spot that I would like to address concerns chromatin remodeling and epigenetic regulation of sperm functions. Because $\mathrm{CB}_{1}$ activation plays a pivotal role in spermiogenesis (that is the developmental stage where DNA is remodeled), it has been recently hypothesized that regulation of the $\mathrm{CB}_{1}$ gene (Cnrl) might also influence chromatin quality in sperm (22). By using Cnrl null mutant ( $\mathrm{Cnrl}^{-/-}$) mice, $\mathrm{CB}_{1}$ activation was demonstrated to regulate indeed chromatin remodeling of spermatids, via either increasing the levels of the Tnp2 gene (encoding for the transition protein 2, that stimulates DNA nick repair in vitro), or enhancing histone displacement. Comparative analysis of wildtype, $\mathrm{Cnrl}^{+/-}$and $\mathrm{Cnr1}^{-/-}$animals suggested the possible occurrence of haploinsufficiency for Tnp2 turnover under $\mathrm{CB}_{1}$ control, whereas histone displacement was disrupted in $\mathrm{CnrI}^{+/-}$and $\mathrm{CnrI}^{-/-}$mice to a lesser extent. Furthermore, flow cytometry analysis demonstrated that the genetic loss of Cnrl decreased sperm chromatin quality and was associated with sperm DNA fragmentation. Of note, this damage increased during epididymal transit, from caput to cauda (22). Collectively, these results demonstrate that the expression (and expectedly the activity) of $\mathrm{CB}_{1}$ controls the physiological alterations of DNA packaging during spermiogenesis and epididymal transit, which might have major implications for male fertility, given the deleterious effects of sperm DNA damage (22). On a final note, it should be recalled that the epigenetic regulation of target genes by eCBs, and conversely that of ECS genes (in particular $\mathrm{CB}_{1}$ ) by pathological conditions, are emerging as a major issue to understand the fine tuning of $\mathrm{eCB}$ signaling in human health and disease (23). Therefore, it can be anticipated that epigenetic studies on sperm quality and fertilizing capacity will open new avenues for preventing or curing (e.g., through a correct lifestyle) human infertility with innovative therapeutics.

In conclusion, distinct ECS elements like $\mathrm{CB}_{1}$ and TRPV1, along with the endogenous levels of their common ligand AEA, hold the promise to represent useful diagnostic biomarkers and therapeutic targets of male fertility defects. It seems noteworthy that, while $\mathrm{CB}_{1}$ has major effects also on female reproductive events (from oocyte development, to ovarian transport, and embryo implantation), apparently TRPV1 does not impact on female fertility (24), apart from generating hyperalgesia via primary sensory neurons during endometriosis (25). Therefore, the latter ion channel seems to represent an ideal target to specifically combat reproductive dysfunctions in males.

\section{ACKNOWLEDGMENTS}

Financial support from the Italian Ministero dell'Istruzione, dell'Università e della Ricerca (PRIN 2010-2011 grant), and from Fondazione TERCAS (grant 2009-2012) is gratefully acknowledged.

\section{REFERENCES}

1. Lewis SE, Maccarrone M. Endocannabinoids, sperm biology and human fertility. Pharmacol Res (2009) 60:126-31. doi:10.1016/j.phrs.2009.02.009

2. Schuel H, Burkman LJ, Lippes J, Crickard K, Forester E, Piomelli D, et al. $N$-Acylethanolamines in human reproductive fluids. Chem Phys Lipids (2002) 121:211-27. doi:10.1016/S0009-3084(02) 00158-5

3. Wang H, Dey SK, Maccarrone M. Jekyll and hyde: two faces of cannabinoid signaling in male and female fertility. Endocr Rev (2006) 27:427-48. doi: 10.1210/er.2006-0006

4. Maccarrone M. Endocannabinoids: friends and foes of reproduction. Prog Lipid Res (2009) 48:344-54. doi:10.1016/j.plipres.2009.07.001

5. Bari M, Battista N, Pirazzi V, Maccarrone M. The manifold actions of endocannabinoids on female and male reproductive events. Front Biosci (2011) 16:498-416. doi:10.2741/3701

6. Di Marzo V, De Petrocellis L. Endocannabinoids as regulators of transient receptor potential (TRP) channels: a further opportunity to develop new endocannabinoid-based therapeutic drugs. Curr Med Chem (2010) 17:1430-49. doi:10.2174/ 092986710790980078

7. Barbonetti A, Vassallo MR, Fortunato D, Francavilla S, Maccarrone M, Francavilla F. Energetic metabolism and human sperm motility: impact of $\mathrm{CB}_{1}$ receptor activation. Endocrinology (2010) 151:5882-92. doi:10.1210/en.2010-0484

8. Bénard G, Massa F, Puente N, Lourenço J, Bellocchio L, Soria-Gómez E, et al. Mitochondrial $\mathrm{CB}_{1}$ receptors regulate neuronal energy metabolism. Nat Neurosci (2012) 15:558-64. doi:10.1038/ nn.3053

9. Morozov YM, Dominguez MH, Varela L, Shanabrough M, Koch M, Horvath TL, et al. Antibodies to cannabinoid type 1 receptor coreact with stomatin-like protein 2 in mouse brain mitochondria. Eur J Neurosci (2013) 38:2341-8. doi:10.1111/ejn.12237

10. Francavilla F, Battista N, Barbonetti A, Vassallo MR, Rapino C, Antonangelo C, et al. Characterization of the endocannabinoid system in human spermatozoa and involvement of transient receptor potential vanilloid 1 receptor in their fertilizing ability. Endocrinology (2009) 150:4692-700. doi:10.1210/en.2009-0057

11. Lewis SE, Rapino C, Di Tommaso M, Pucci M, Battista N, Paro R, et al. Differences in the endocannabinoid system of sperm from fertile and infertile men. PLoS One (2012) 7:e47704. doi:10. 1371/journal.pone.0047704

12. Amoako AA, Marczylo TH, Marczylo EL, Elson J, Willets JM, Taylor AH, et al. Anandamide modulates human sperm motility: implications for men 
with asthenozoospermia and oligoasthenoteratozoospermia. Hum Reprod (2013) 28:2058-66. doi: 10.1093/humrep/det232

13. Flesch FM, Brouwers JF, Nievelstein PF, Verkleij AJ, van Golde LM, Colenbrander B, et al. Bicarbonate stimulated phospholipid scrambling induces cholesterol redistribution and enables cholesterol depletion in the sperm plasma membrane. J Cell Sci (2001) 114(19):3543-55.

14. Miranda PV, Allaire A, Sosnik J, Visconti PE. Localization of low-density detergent-resistant membrane proteins in intact and acrosome-reacted mouse sperm. Biol Reprod (2009) 80:897-904. doi:10.1095/biolreprod.108.075242

15. Botto L, Bernabò N, Palestini P, Barboni B. Bicarbonate induces membrane reorganization and CBR1 and TRPV1 endocannabinoid receptor migration in lipid microdomains in capacitating boar spermatozoa. J Membr Biol (2010) 238:33-41. doi:10.1007/s00232-010-9316-8

16. Dainese E, Oddi S, Maccarrone M. Interaction of endocannabinoid receptors with biological membranes. Curr Med Chem (2010) 17:1487-99. doi: 10.2174/092986710790980087

17. Ambrosini A, Zolese G, Wozniak M, Genga D, Boscaro M, Mantero F, et al. Idiopathic infertility: susceptibility of spermatozoa to in-vitro capacitation, in the presence and the absence of palmitylethanolamide (a homologue of anandamide), is strongly correlated with membrane polarity studied by Laurdan fluorescence. $\mathrm{Mol}$
Hum Reprod (2003) 9:381-8. doi:10.1093/molehr/ gag056

18. Ambrosini A, Zolese G, Ambrosi S, Bertoli E, Mantero $\mathrm{F}$, Boscaro $\mathrm{M}$, et al. Idiopathic infertility: effect of palmitoylethanolamide (a homologue of anandamide) on hyperactivated sperm cell motility and $\mathrm{Ca}^{2+}$ influx. J Androl (2005) 26:429-36. doi:10.2164/jandrol.04141

19. Bian X, Gandahi JA, Liu Y, Yang P, Liu Y, Zhang $\mathrm{L}$, et al. The ultrastructural characteristics of the spermatozoa stored in the cauda epididymidis in Chinese soft-shelled turtle Pelodiscus sinensis during the breeding season. Micron (2013) 44:202-9. doi:10.1016/j.micron.2012.06.010

20. Oddi S, Fezza F, Pasquariello N, De Simone C, Rapino C, Dainese E, et al. Evidence for the intracellular accumulation of anandamide in adiposomes. Cell Mol Life Sci (2008) 65:840-50. doi:10. 1007/s00018-008-7494-7

21. Maccarrone M, Dainese E, Oddi S. Intracellular trafficking of anandamide: new concepts for signaling. Trends Biochem Sci (2010) 35:601-8. doi:10.1016/j.tibs.2010.05.008

22. Chioccarelli T, Cacciola G, Altucci L, Lewis SE, Simon L, Ricci G, et al. Cannabinoid receptor 1 influences chromatin remodeling in mouse spermatids by affecting content of transition protein 2 mRNA and histone displacement. Endocrinology (2010) 151:5017-29. doi:10.1210/en.2010-0133

23. D’Addario C, Di Francesco A, Pucci M, Finazzi Agrò A, Maccarrone M. Epigenetic mechanisms and endocannabinoid signalling. FEBS $J$ (2013) 280:1905-17. doi:10.1111/febs.12125

24. Battista N, Meccariello R, Cobellis G, Fasano $\mathrm{S}$, Di Tommaso M, Pirazzi V, et al. The role of endocannabinoids in gonadal function and fertility along the evolutionary axis. Mol Cell Endocrinol (2012) 355:1-14. doi:10.1016/j.mce. 2012.01.014

25. Liu J, Liu X, Duan K, Zhang Y, Guo SW. The expression and functionality of transient receptor potential vanilloid 1 in ovarian endometriomas. Reprod Sci (2012) 19:1110-24. doi:10.1177/ 1933719112443876

Received: 24 October 2013; accepted: 24 October 2013; published online: 08 November 2013.

Citation: Maccarrone M (2013) Endocannabinoids as markers of sperm quality: hot spots. Front. Endocrinol. 4:169. doi: 10.3389/fendo.2013.00169

This article was submitted to Experimental Endocrinology, a section of the journal Frontiers in Endocrinology. Copyright (C) 2013 Maccarrone. This is an open-access article distributed under the terms of the Creative Commons Attribution License (CC BY). The use, distribution or reproduction in other forums is permitted, provided the original author(s) or licensor are credited and that the original publication in this journal is cited, in accordance with accepted academic practice. No use, distribution or reproduction is permitted which does not comply with these terms. 